\title{
Zofenopril Plus Hydrochlorothiazide and Irbesartan Plus Hydrochlorothiazide in Previously Treated and Uncontrolled Diabetic and Non-diabetic Essential Hypertensive Patients
}

\author{
Enrico Agabiti-Rosei • Athanasios Manolis •
}

Dario Zava $\cdot$ Stefano Omboni

To view enhanced content go to www.advancesintherapy.com Received: October 30, 2013 / Published online: January 11, 2014

(c) The Author(s) 2014. This article is published with open access at Springerlink.com

\section{ABSTRACT}

Introduction: In most treated patients with hypertension, a two or more drug combination is required to achieve adequate blood pressure (BP) control. In our study we assessed whether the combination of zofenopril + hydrochlorothiazide (HCTZ) was at least as effective as irbesartan + HCTZ in essential hypertensives with at least one

On behalf of the ZODIAC Study Group.

Trial registration: EudraCT number: 2008-002439-3.

Electronic supplementary material The online version of this article (doi:10.1007/s12325-013-0090-8) contains supplementary material, which is available to authorized users.

E. Agabiti-Rosei

Division of Medicine and Surgery, Spedali Civili and University of Brescia, Brescia, Italy

A. Manolis

Asklepeion General Hospital of Voula, University of Athens, Athens, Greece

D. Zava

Istituto Lusofarmaco d'Italia, Milan, Italy

S. Omboni ( $₫)$

Italian Institute of Telemedicine, Varese, Italy

e-mail: stefano.omboni@iitelemed.org additional cardiovascular risk factor, uncontrolled by a previous monotherapy.

Methods: After a 2-week placebo washout, 361 treated hypertensive patients [office sitting diastolic BP (DBP), $\quad \geq 90 \mathrm{mmHg}]$, aged 18-75 years, were randomized double blind to 18-week treatment with zofenopril $30 \mathrm{mg}$ plus HCTZ $12.5 \mathrm{mg}$ or irbesartan $150 \mathrm{mg}$ plus HCTZ $12.5 \mathrm{mg}$ once daily, in an international, multicenter study. After the first 6 and 12 weeks, zofenopril and irbesartan doses could be doubled in non-normalized subjects. The primary study end point was the office sitting DBP reduction after 18 weeks of treatment. Secondary end points included office systolic BP (SBP), ambulatory BP and high sensitivity C-reactive protein (hs-CRP).

Results: The between-treatment difference for office DBP averaged to $+1.0(95 \% \mathrm{CI}-0.4,+0.8)$ mmHg $(P=0.150)$, the upper limit of the $95 \%$ confidence interval being inferior to the protocol-defined non-inferiority limit ( $3 \mathrm{mmHg}$ ). In the subset of patients with valid ambulatory BP, no difference in 24-h average DBP $[n=181 ; 6.7(8.7,4.6)$ zofenopril + HCTZ vs. $6.3(8.8,3.7) \mathrm{mmHg}$ irbesartan $+\mathrm{HCTZ}$, $P=0.810]$ and SBP reductions $[11.7(15.4,8.0)$ 
vs. $12.6(17.2,8.0) \mathrm{mmHg}, P=0.758$ ] were observed between the two treatment groups. hs-CRP was reduced by zofenopril + HCTZ $[-0.52(-1.05,0.01) \mathrm{mg} / \mathrm{L}]$, while it was increased by irbesartan plus HCTZ [0.97 (0.29, 1.65) $\mathrm{mg} / \mathrm{L}, P=0.001$ between treatments].

Conclusion: In previously monotherapy-treated, uncontrolled patients with hypertension, zofenopril $30-60 \mathrm{mg}+\mathrm{HCTZ} 12.5 \mathrm{mg}$ is as effective as irbesartan 150-300 mg plus HCTZ $12.5 \mathrm{mg}$, with the added value of a potential protective effect against vascular inflammation.

Keywords: Ambulatory blood pressure monitoring; Cardiology; Essential hypertension; Hydrochlorothiazide; Irbesartan; Office blood pressure; Zofenopril

\section{INTRODUCTION}

Arterial hypertension affects almost 30\% of the adult population worldwide [1] and is currently considered as a major risk factor for an array of cardiovascular and related diseases [2]. Numerous randomized, placebo-controlled studies have conclusively demonstrated that in patients with hypertension, blood pressure (BP) reduction lowers the incidence of cardiovascular morbid and fatal events $[3,4]$.

Large pharmacological trials have also documented that combination therapy with two or more drugs is required to achieve BP control in most patients with hypertension, particularly in those with associated cardiovascular risk factors or at high risk for cardiovascular events [5-8]. For these reasons, guidelines on the management of hypertension currently recommend the use of two drug combinations as a first-line therapy $[9,10]$.

One of the most effective two drug antihypertensive combinations is that between an angiotensin converting enzyme (ACE) inhibitor or an angiotensin II receptor blocker (ARB) and a diuretic, in which the ACE inhibitor and the ARB antagonize the counter-regulatory system activity triggered by the diuretic, thus improving the efficacy and tolerability of singledrug components [11].

Zofenopril calcium, a pro-drug of the active compound zofenoprilat, is a highly lipophilic ACE inhibitor which has been successfully and safely employed in the treatment of essential hypertension [12] and acute myocardial infarction or heart failure [13] and also in subgroups of patients with elevated BP [14, 15]. In subjects with essential hypertension, zofenopril has been shown to be as effective as atenolol [16], hydrochlorothiazide (HCTZ) [17], amlodipine [18], enalapril [19], lisinopril [20], losartan [21], and candesartan [22].

Irbesartan is an ARB, characterized by a high bioavailability, a long duration of action, and a small potential for pharmacological interactions [23]. The drug has showed a high efficacy in lowering BP in hypertensive patients, particularly those with renal impairment, where it demonstrated the most remarkable evidence of efficacy within the ARB class [24]. Comparative clinical trials performed in mild-to-moderate hypertension showed equal efficacy, but better tolerability of irbesartan, compared to the other major antihypertensive classes, including beta-blockers (atenolol), calcium antagonists (amlodipine), ACE inhibitors (enalapril), and renin inhibitors (aliskiren), and superior efficacy as compared to doxazosin $[23,24]$.

Both zofenopril and irbesartan have been also successfully employed in hypertensive patients in combination with a diuretic [23-26]. However, since direct comparative data on the antihypertensive efficacy and safety of this two drug combination are lacking and since most studies did not test the 
efficacy of the highest available dose of zofenopril $(60 \mathrm{mg}$ ) plus HCTZ, the present study was devised. Irbesartan was selected as comparator, because its efficacy was shown to be superior to that of other common ARBs such as losartan and valsartan [23, 24]. To comply with the current recommendations, the patients targeted for treatment were those with an essential hypertension not controlled by a previous monotherapy associated with one or more additional cardiovascular risk factors. To make the comparison particularly stringent, efficacy assessment was based not only on conventional office BP measurements taken $24 \mathrm{~h}$ post-dosing, but also on ambulatory monitoring over $24 \mathrm{~h}$.

\section{METHODS}

\section{Study Population}

Essential hypertension patients (sitting office diastolic blood pressure (DBP) $\geq 90 \mathrm{mmHg}$ ) of both genders, aged 18-75 years, with at least one additional cardiovascular risk factor, uncontrolled by previous monotherapy, were eligible for study participation. The following cardiovascular risk factors were considered among the inclusion criteria [9]: (a) current smoking; (b) elevated total cholesterol (>190 mg/dL) or specific lipid-lowering drug treatment; (c) elevated low density lipoprotein (LDL) cholesterol (>115 mg/dL) or specific lipidlowering drug treatment; (d) low high density lipoprotein (HDL) cholesterol $(<40 \mathrm{mg} / \mathrm{dL}$ in males and $<46 \mathrm{mg} / \mathrm{dL}$ in females) or specific lipid-lowering drug treatment; (e) diabetes mellitus controlled by diet or specific antidiabetic therapy (HbA1c $\leq 7.5 \%$ ); (f) abdominal obesity: waist circumference $<102 \mathrm{~cm}$ in males and $<88 \mathrm{~cm}$ in females, or body mass index (BMI) between 25 and $32 \mathrm{~kg} / \mathrm{m}^{2}$; and (g) family history of premature cardiovascular disease (males at age $<55$ years and females at age $<65$ years).

Patients were excluded if they had: (a) secondary or malignant hypertension; (b) isolated systolic hypertension; (c) orthostatic hypotension [office systolic blood pressure (SBP), drop upon standing $\geq 20 \mathrm{mmHg}$; (d) history of heart failure requiring medical treatment; (e) myocardial infarction or cerebrovascular accidents in the previous 6 months; (f) hemodynamically significant cardiac valve disease; (g) severe or clinically significant systemic, renal, hepatic, neurological, or psychiatric disease; (h) moderate-severe obesity $\left(\mathrm{BMI}>32 \mathrm{~kg} / \mathrm{m}^{2}\right)$; (i) large (circumference $>32 \mathrm{~cm}$ ) or tiny upper arm (circumference $<24 \mathrm{~cm}$ ); and (j) known hypersensitivity to ACE inhibitors, ARBs, or thiazide diuretics.

Pregnant women and breast-feeding mothers were excluded as well. Women with childbearing potential or within 2 years from menopause had to practice an effective method of birth control and were required to have a negative urine pregnancy test.

The study was conducted according to Good Clinical Practice guidelines and the protocol was approved by the ethics committees of the centers involved. All procedures followed were in accordance with the ethical standards of the responsible committee on human experimentation (institutional and national) and with the Helsinki Declaration of 1975, as revised in 2000 and 2008. Informed consent was obtained from all patients for being included in the study.

\section{Study Design}

This was an international, multicenter, randomized (1:1), double-blind, parallel group study conducted at 27 hospitals located in five 
different European countries: seven centers in Italy, three in Greece, three in Lithuania, four in Romania, and ten in Turkey. The study consisted of a 2-week single-blind run-in period during which previous antihypertensive treatment was stopped and replaced with placebo, followed by 18 weeks of double-blind treatment with zofenopril or irbesartan at the initial doses of 30 or $150 \mathrm{mg}$ combined with HCTZ $12.5 \mathrm{mg}$. Placebo and study drugs were given orally and once daily (between 9 and 11 a.m.) with a glass of water. After the first 6 and 12 weeks of active treatment, the dose of zofenopril or ramipril had to be doubled, respectively, to 60 and $300 \mathrm{mg}$, if office SBP was $\geq 140 \mathrm{mmHg}$ or office DBP was $\geq 90 \mathrm{mmHg}$ in non-diabetic patients and if office SBP was $\geq 130 \mathrm{mmHg}$ or office DBP was $\geq 80 \mathrm{mmHg}$ in diabetic patients [27].

At the screening visit, informed consent was obtained and medical history collected. At the same visit, physical examination, a 12-lead electrocardiography (ECG), BP and heart rate measurements and laboratory tests (blood count, glucose, total, LDL and HDL cholesterol, triglycerides, uric acid, creatinine, sodium and potassium, transaminases and $\gamma$-GT, total bilirubin, $\mathrm{HBA}_{1 \mathrm{c}}$, high sensitivity C-reactive protein (hs-CRP), urinalysis, and urine pregnancy tests) were carried out locally. No centralized assessment of laboratory tests was foreseen. Physical examination and BP and heart rate measurements were repeated at each visit $(6,12$, and 18 weeks after randomization), while an ECG was assessed again and laboratory tests checked at the end of the 18 weeks of double-blind treatment. Adverse events, assumption of concomitant medications, and compliance to treatment were assessed at each visit. At the end of the placebo run-in period and 18 weeks of double-blind treatment, BP was measured by 24 -h ambulatory monitoring.

\section{Office Blood Pressure and Heart Rate Measurement}

Blood pressure and heart rate were measured in the office by a validated, automatic, electronic, upper arm sphygmomanometer (A\&D UA767PC, A\&D Company Limited, Tokyo, Japan) [28], approximately $24 \mathrm{~h}$ after the last placebo or drug intake. The arm cuff was kept at the heart level during every BP measurement. Three measurements, taken at 2-min intervals, after 5 min of rest in the sitting position were averaged and used as the office BP reference value. Blood pressure and heart rate values were also taken after 1 and 4 min of standing.

\section{Ambulatory Blood Pressure Measurements}

Ambulatory BP monitoring was performed at randomization and the final visit, noninvasively, over $24 \mathrm{~h}$ by an oscillometric, validated, automatic, electronic device (A\&D TM-2430, A\&D Company Limited, Tokyo, Japan) [29]. The monitoring cuff was wrapped around the non-dominant arm and the patient was asked to keep her/his arm still during the automatic BP measurements. The device was programmed to measure BP every $15 \mathrm{~min}$ throughout the whole monitoring period. Each recording started in the morning, immediately after office BP assessment and administration of placebo or active treatment. Patients were then sent home, asked to resume normal life and to come back $24 \mathrm{~h}$ later for removal of the device. Results of the recording were read by connecting the BP measuring device to a wireless interface which sent data to a centralized data management center (Central Core Laboratory, Biotechmed, Varese, Italy) through the mobile telephone network and the web [30]. Traces had to be analyzed real time and in case of a bad-quality recording (see 
below) the investigator was contacted by the central core laboratory to repeat the recording in the next 2 days, whenever possible.

\section{Data Analysis}

The primary efficacy end point of the study was the between-treatment comparison of sitting office DBP changes from baseline to the end of the 18 weeks of double-blind treatment.

This was a non-inferiority trial; thus the hypothesis was that zofenopril plus HCTZ had to be defined as being non-inferior to irbesartan plus HCTZ in case of a difference within $3 \mathrm{mmHg}$ for DBP, with a common standard deviation of $9 \mathrm{mmHg}$. Using a one-tailed $t$ test with a 0.025 significance level and an $80 \%$ power, the estimated number of patients to be randomized was 320 (including a 10\% dropout rate), with 160 for each treatment group.

Analysis was performed on patients valid for intention to treat, defined as all randomized patients receiving at least one dose of active treatment drug and having at least one office BP measurement after randomization. The lastobservation-carried-forward method was used for patients prematurely leaving the study. The per-protocol population included all randomized patients completing the 18-week double-blind study period without major protocol violations and was used for confirmatory analysis.

Secondary study end points were betweentreatment comparison of: (a) office sitting SBP changes after 18 weeks of double-blind treatment; (b) the percentage of patients with a sitting office SBP $<140 \mathrm{mmHg}$ and DBP $<90$ mmHg after 18 weeks of double-blind treatment; (c) the percentage of patients with a sitting office SBP $<130 \mathrm{mmHg}$ and DBP $<80 \mathrm{mmHg}$ after 18 weeks of double-blind treatment; (d) the percentage of normalized (sitting office SBP
$<140 \mathrm{mmHg}$ and DBP $<90 \mathrm{mmHg}$ ) plus responder patients (sitting office SBP reduction $\geq 20 \mathrm{mmHg}$ or DBP reduction $\geq 10 \mathrm{mmHg}$ ) after 18 weeks of double-blind treatment; (e) changes in 24-h average SBP and DBP after 18 weeks of treatment; (f) hourly averages of SBP and DBP before and during treatment; (g) SBP and DBP changes in the last $6 \mathrm{~h}$ of the dosing interval after 18 weeks of treatment; (h) smoothness index of SBP and DBP after 18 weeks of treatment [31].

The analysis of 24-h BP recordings was preceded by removal of artifacts according to previously described editing criteria [32]. Recordings were considered valid when no more than $1 \mathrm{~h}$ was missing over the $24 \mathrm{~h}$ and when at least $70 \%$ of the expected measurements were available.

Safety analysis was applied to all randomized patients, by calculating the incidence of adverse events and changes in laboratory data or ECG during the study.

Between-treatment differences in mean sitting office DBP changes at week 18 were assessed by analysis of covariance, by adjusting for the baseline value and considering the center effect. Given the unbalanced distribution of cardiovascular risk factors between the two randomization groups, an analysis was run taking into account the baseline value, the country effect, and the presence of at least one cardiovascular risk factor among advanced age, increased waist circumference, current smoking, alcohol drinking, and diabetes. The latter analysis was applied to all the secondary end points. Comparison of normalized and normalized plus responder patients between the two treatment groups was performed by the Chisquare test. Subgroup analysis by drug doses, type of hypertension (mild or moderate), and presence of diabetes was also made. The level of 
statistical significance was kept at 0.05 throughout the whole study. Data are shown as mean $\pm \mathrm{SD}$, mean and $95 \%$ confidence interval, and absolute (n) or relative (\%) frequency.

All data analysis was performed using SAS Software Version 9.2 (SAS Institute Inc., NC, USA).

\section{RESULTS}

\section{Baseline Demographic and Clinical Data}

A total of 408 patients were screened, but 47 were lost during the placebo washout period. Thus the number of patients randomized to one of the two treatment arms was 361. Of these patients 327 completed the 18-week doubleblind randomized phase, while 34 discontinued the study because of consent withdrawal $(n=16)$, lack of compliance to study procedures $(n=7)$, adverse events $(n=7)$, lack of efficacy $(n=1)$, protocol violation $(n=2)$, or being lost to follow-up $(n=1)$. A flowchart of the patients throughout the study is presented in Fig. 1.

Overall, 353 patients were eligible for the intention-to-treat analysis (175 in the zofenopril plus HCTZ and 178 in the irbesartan plus HCTZ treatment group) and 294 for the per-protocol analysis (152 in the zofenopril plus HCTZ and 142 in the irbesartan plus HCTZ treatment group). 181 out of 356 patients undergoing ambulatory BP monitoring at baseline had valid recordings and were included in this subgroup analysis (95 randomized to zofenopril plus $\mathrm{HCTZ}$ and 86 to irbesartan plus HCTZ).

As shown in Table 1, patients randomized to zofenopril plus HCTZ displayed a higher cardiovascular risk profile with respect to those randomized to irbesartan plus HCTZ: they were older, made a broader use of concomitant therapies, and showed a larger prevalence of additional cardiovascular risk factors besides hypertension.

The subgroup of patients undergoing a 24-h BP monitoring and randomized to zofenopril plus HCTZ was older than that assigned to the irbesartan plus HCTZ group ( $58 \pm 9$ vs. $54 \pm 10$ years, $P=0.005$ ), while no between-treatment differences were observed for other demographic and clinical data. In the ambulatory BP monitoring subgroup, entry office SBP and DBP values did not differ between the two treatment arms $(151 \pm 13 / 96 \pm 5 \mathrm{mmHg}$ zofenopril plus HCTZ and $151 \pm 11 / 97 \pm 5 \mathrm{mmHg}$ irbesartan plus HCTZ, $P=0.780$ for SBP and $P=0.255$ for DBP).

\section{Drug Dosing}

The 60-mg dose of zofenopril in combination with HCTZ $12.5 \mathrm{mg}$ was taken at the end of the study by $68.6 \%$ of patients randomized to this drug and the 300-mg dose of irbesartan (plus HCTZ $12.5 \mathrm{mg}$ ) by $61.2 \%$ of the patients $(P=0.183)$. The proportion of patients under full drug dose did not differ in the subgroup of obese patients $(77.8 \%$ zofenopril vs. 64.9 irbesartan, $P=0.135)$.

In the ambulatory BP monitoring subgroup, the proportion of patients taking a higher dose of both drugs was in line with the figure for the main study population $(72.6 \%$ zofenopril vs. $65.1 \%$ irbesartan, $P=0.275)$.

\section{Sitting Office Blood Pressure}

The between-treatment difference for office DBP (primary end point) averaged to +1.0 


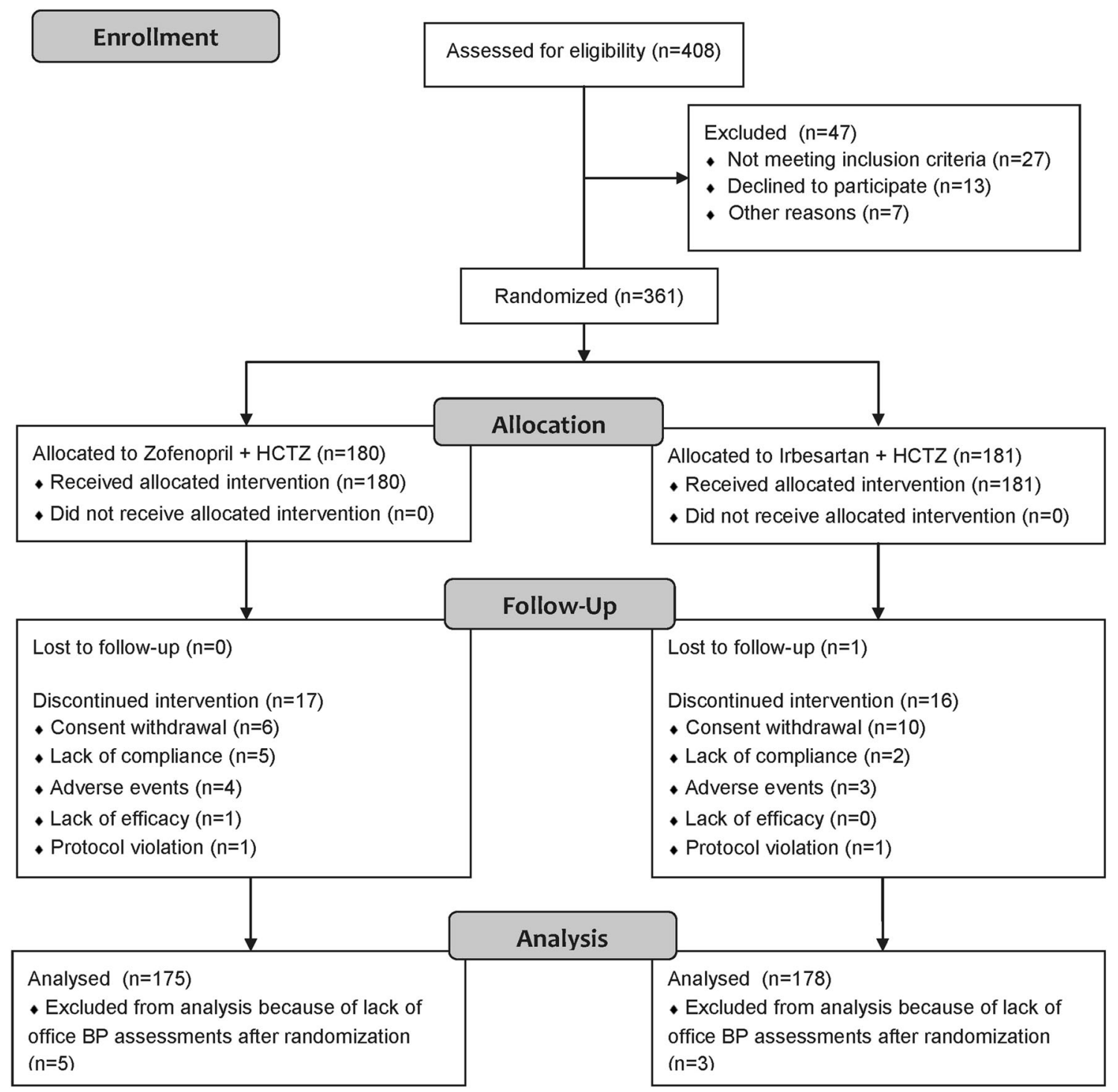

Fig. 1 Flow diagram of the patients through the different phases of the study

(95\% CI $-0.4,+0.8) \mathrm{mmHg}(P=0.150)$, with the upper limit of the $95 \%$ confidence interval being inferior to the protocol-defined noninferiority limit of $3 \mathrm{mmHg}$.

Figure 2 shows baseline-adjusted office sitting DBP and SBP reductions in the intention-to-treat population after correction for the country effect and type of cardiovascular risk factors. Office BP values were progressively and significantly $(P<0.01)$ reduced by both treatment regimens during the study. At the final evaluation (week 18), mean sitting office DBP reduction (and 95\% confidence interval) achieved with zofenopril combined with the diuretic was 17.6 (19.9, $15.3)$ vs. $15.1 \quad(17.5,12.6) \quad \mathrm{mmHg}$ with irbesartan plus HCTZ, showing a difference of $-2.6(-5.9,+0.8) \mathrm{mmHg}(P=0.134)$ between the two drug treatments. Office sitting SBP reductions at the end of the study were 21.5 $(24.5,18.6) \mathrm{mmHg}$ in the zofenopril and 20.6 $(24.1,17.2) \mathrm{mmHg}$ in the irbesartan treatment 
Table 1 Demographic and clinical data of the patients of the intention-to-treat population at the time of randomization $(n=353)$

\begin{tabular}{|c|c|c|c|}
\hline Baseline characteristics & $\begin{array}{l}\text { Zofenopril 30-60 mg }+ \\
\text { HCTZ } 12.5 \mathrm{mg}(n=175)\end{array}$ & $\begin{array}{l}\text { Irbesartan } 150-300 \mathrm{mg}+ \\
\text { HCTZ } 12.5 \mathrm{mg}(n=178)\end{array}$ & $P$ value \\
\hline Age (years, mean $\pm S D)$ & $56 \pm 11$ & $54 \pm 11$ & 0.024 \\
\hline Males $(n, \%)$ & $112(64)$ & $99(56)$ & 0.108 \\
\hline $\mathrm{BMI}\left(\mathrm{kg} / \mathrm{m}^{2}\right.$, mean $\left.\pm \mathrm{SD}\right)$ & $28 \pm 3$ & $28 \pm 3$ & 0.357 \\
\hline \multicolumn{4}{|l|}{ BMI class $(n, \%)\left(\mathrm{kg} / \mathrm{m}^{2}\right)$} \\
\hline$<25$ & $32(18)$ & $33(19)$ & \multirow[t]{3}{*}{0.962} \\
\hline $25-29.9$ & $89(51)$ & $88(49)$ & \\
\hline$\geq 30$ & $54(31)$ & $57(32)$ & \\
\hline Waist circumference $(\mathrm{cm}$, mean $\pm \mathrm{SD})$ & $100 \pm 10$ & $97 \pm 10$ & 0.023 \\
\hline Concomitant diseases $(n, \%)$ & $151(86)$ & $144(81)$ & 0.172 \\
\hline Concomitant therapies $(n, \%)$ & $102(58)$ & $79(44)$ & 0.009 \\
\hline \multicolumn{4}{|c|}{ Type of previous antihypertensive treatment $(n, \%)$} \\
\hline ACE inhibitors & $68(39)$ & $54(30)$ & \multirow[t]{4}{*}{0.221} \\
\hline $\mathrm{ARBs}$ & $47(27)$ & $58(33)$ & \\
\hline Calcium-channel blockers & $37(21)$ & $41(23)$ & \\
\hline Others & $23(13)$ & $25(14)$ & \\
\hline Diabetes $(n, \%)$ & $34(19)$ & $29(16)$ & 0.442 \\
\hline Alcohol drinking $(n, \%)$ & $81(46)$ & $74(42)$ & 0.372 \\
\hline Cigarette smoking $(n, \%)$ & $56(32)$ & $45(25)$ & 0.163 \\
\hline Other CV risk factors $(n, \%)$ & $143(82)$ & $129(73)$ & 0.039 \\
\hline Sitting office SBP (mmHg) & $152 \pm 13$ & $151 \pm 12$ & 0.549 \\
\hline Sitting office DBP (mmHg) & $96 \pm 5$ & $96 \pm 5$ & 0.728 \\
\hline
\end{tabular}

Data are separately shown for the two groups of randomization and reported as mean $( \pm \mathrm{SD})$ or absolute $(n)$ and relative frequency (\%). The $P$ value refers to the statistical significance of between-treatment differences

$A C E$ angiotensin converting enzyme, $A R B$ angiotensin II receptor blocker, $B M I$ body mass index, $C V$ cardiovascular, $D B P$ diastolic blood pressure, $H C T Z$ hydrochlorothiazide, SBP systolic blood pressure

group, with no between-treatment differences $(P=0.691)$.

At the end of the study, office sitting DBP and SBP reductions were similar with zofenopril $30 \mathrm{mg}$ plus HCTZ [DBP $16.4(18.6,14.3) \mathrm{mmHg}$; SBP $18.2(21.8,14.6) \mathrm{mmHg}]$ and with irbesartan $150 \mathrm{mg}$ plus HCTZ [15.0 (17.4, 12.6) and $22.0(25.9,18.1) \mathrm{mmHg}]$ in the subgroup of patients with diabetes $(P=0.366$ between groups for DBP and $P=0.115$ for SBP).

\section{Normalized and Responder Patients}

Sitting office BP normalization (SBP $<140$ and DBP $<90 \mathrm{mmHg}$ ) was achieved by the end of the 18 weeks of double-blind treatment by a similar 
Table 2 Average 24-h ambulatory diastolic (DBP) and systolic blood pressure (SBP) at randomization and baselineadjusted reductions after 18 weeks of treatment with zofenopril + hydrochlorothiazide (HCTZ) or irbesartan + HCTZ

\begin{tabular}{llll}
\hline Average 24-h BP reduction & $\begin{array}{l}\text { Zofenopril 30-60 mg }+ \\
\text { HCTZ 12.5 } \mathbf{~ m g ~}(\boldsymbol{n}=\mathbf{9 5})\end{array}$ & $\begin{array}{l}\text { Irbesartan 150-300 } \mathbf{~ m g}+ \\
\text { HCTZ 12.5 } \mathbf{~ m g ~}(\boldsymbol{n}=\mathbf{8 6})\end{array}$ & $\boldsymbol{P}$ value \\
\hline 24-h DBP (mmHg) & & & \\
Baseline & $84.8 \pm 8.0$ & $86.1 \pm 7.6$ & 0.810 \\
Reduction with treatment & $6.7(8.7,4.6)$ & $6.3(8.8,3.7)$ & \\
24-h SBP (mmHg) & & $142.2 \pm 13.0$ & 0.758 \\
Baseline & $144.6 \pm 14.0$ & $12.6(17.2,8.0)$ & \\
Reduction with treatment & $11.7(15.4,8.0)$ &
\end{tabular}

Data are shown for the intention-to-treat population and reported as mean and $95 \%$ confidence interval. The $P$ value refers to the statistical significance of the between-treatment difference

$B P$ blood pressure
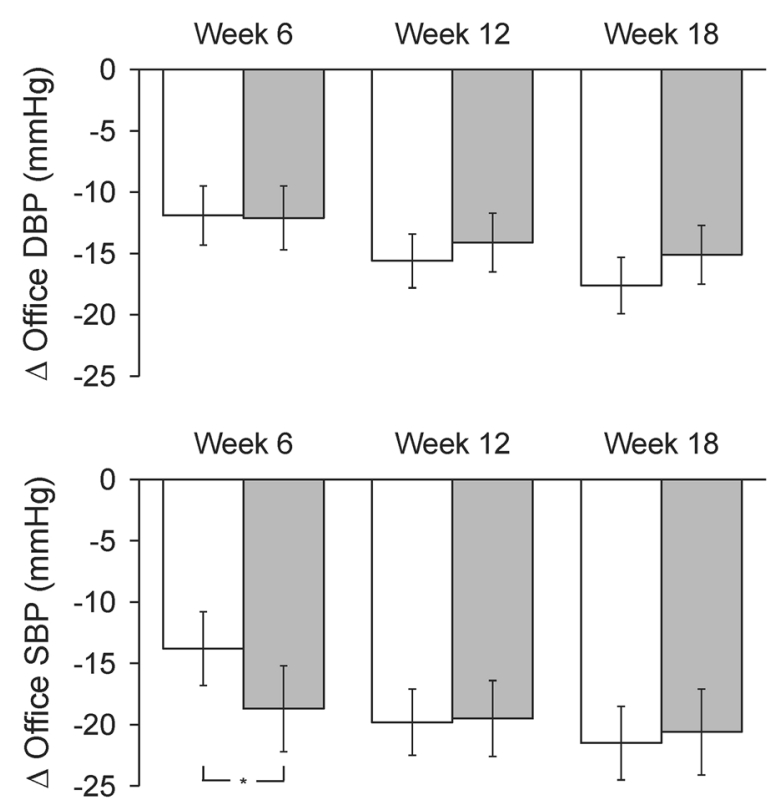

Fig. 2 Baseline-adjusted office sitting diastolic (DBP) and systolic blood pressure (SBP) mean changes (95\% confidence intervals) from baseline after 6, 12, and 18 weeks of treatment with zofenopril $30-60 \mathrm{mg}$ plus hydrochlorothiazide (HCTZ) $12.5 \mathrm{mg} \quad(n=175$, open bars) and irbesartan 150-300 mg plus HCTZ $12.5 \mathrm{mg}$ ( $n=178$, full bars), for the intention-to-treat population. Asterisk refers to the statistical significance of betweentreatment differences $\left({ }^{*} P<0.05\right)$

proportion of patients treated with zofenopril plus HCTZ (79.6\%) and irbesartan plus HCTZ (79.5\%, $P=0.973)$. The rate of normalized patients was lower when a tighter BP goal was considered (SBP $<130$ and DBP $<80 \mathrm{mmHg}$ ): $59.3 \%$ zofenopril plus HCTZ vs. $53.6 \%$ irbesartan plus HCTZ $(P=0.387)$. Finally, the proportion of normalized or responder patients was identical between zofenopril plus the diuretic $(88.4 \%)$ and irbesartan plus the diuretic $(88.5 \% ; P=0.981)$.

The results of the intention-to-treat analysis on office BP changes and normalized and responder rates were confirmed on the perprotocol population (data not shown).

\section{Ambulatory Blood Pressure Monitoring}

Also in the population of patients with valid ambulatory recordings, sitting office DBP and SBP reductions were similar with zofenopril plus HCTZ $[15.4(17.5,13.2)$ and $21.2(24.4,18.0)$ $\mathrm{mmHg}$ ] and irbesartan plus HCTZ [16.8 (19.4, 14.2) and $23.2(27.1 / 19.2) \mathrm{mmHg} ; P=0.397$ for DBP and $P=0.458$ for SBP]. Treatment-induced 24-h DBP and SBP reductions under zofenopril plus HCTZ did not significantly differ from those under irbesartan plus HCTZ (Table 2).

As shown in Fig. 3, both drugs reduced BP during every hour in which the $24 \mathrm{~h}$ were 

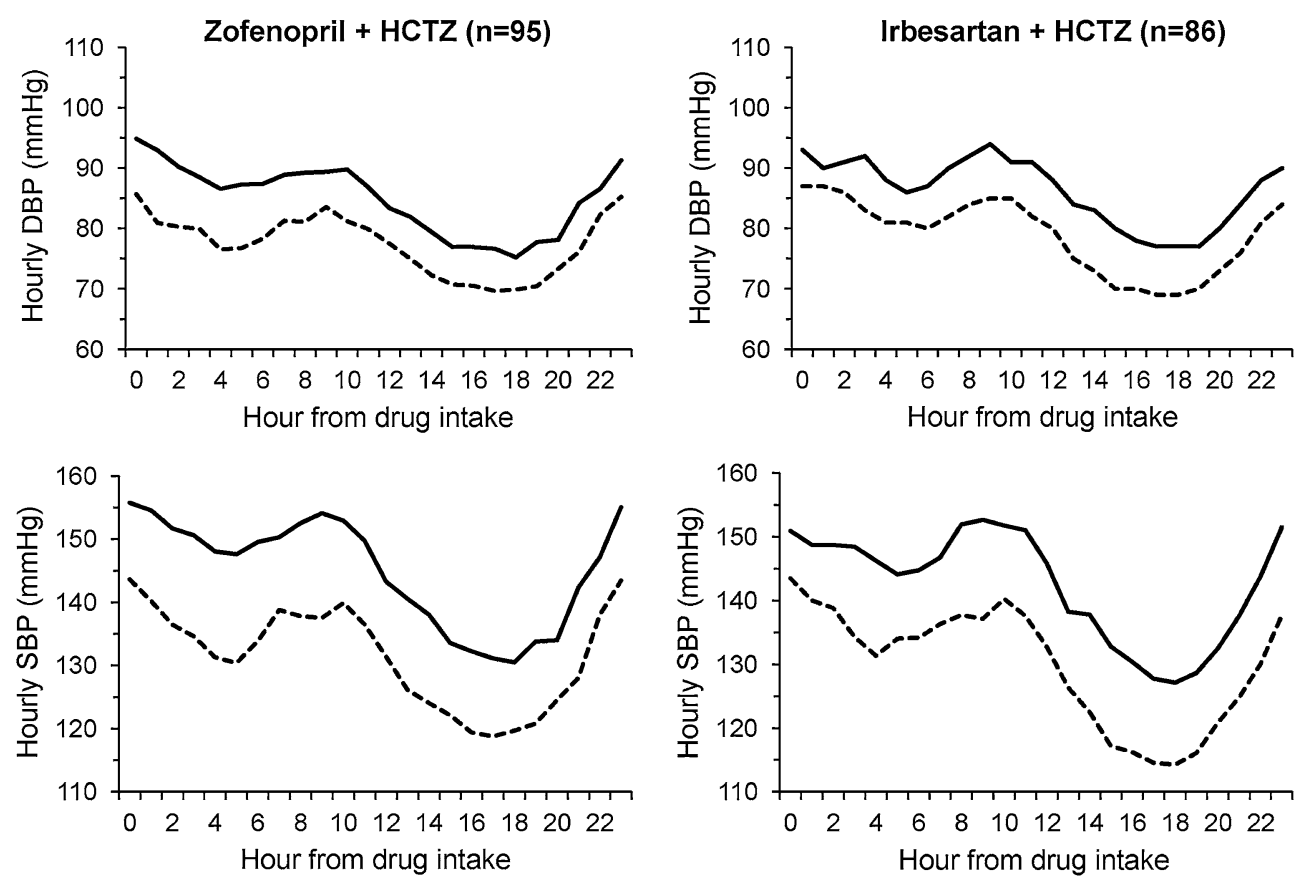

Fig. 3 Average hourly diastolic (DBP) and systolic blood pressure (SBP) values at baseline (continuous line) and at the end of the 18-week double-blind treatment (dashed lines) in patients treated with zofenopril 30-60 mg plus

hydrochlorothiazide (HCTZ) $12.5 \mathrm{mg} \quad(n=95)$ or irbesartan $150-300 \mathrm{mg}$ plus HCTZ $12.5 \mathrm{mg}(n=86)$. Data are shown for the patients of the intention-to-treat population with valid 24 -h recordings $(n=181)$

divided. The drug efficacy in the last 6-h period from the dosing interval, covering the last part of the night sleep and the hours of awakening, was similar for both DBP $[5.6(8.3,3.0)$ vs. 5.7

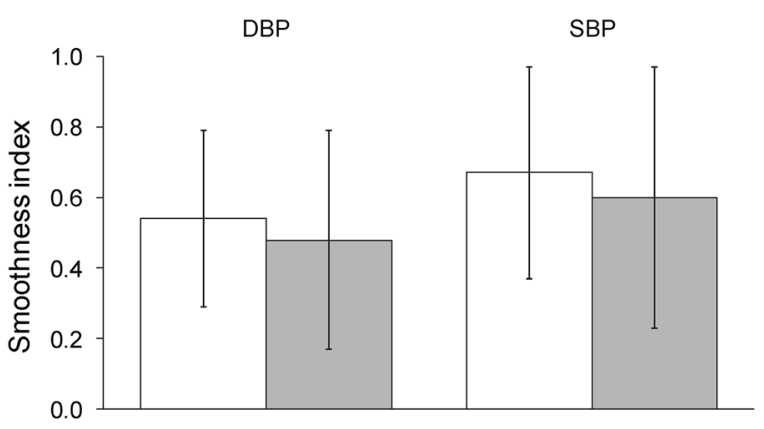

Fig. 4 Average smoothness index $( \pm S D)$ of diastolic (DBP) and systolic blood pressure (SBP) for zofenopril 30-60 mg plus hydrochlorothiazide (HCTZ) $12.5 \mathrm{mg}$ $(n=95$, open bars $)$ and irbesartan $150-300 \mathrm{mg}$ plus HCTZ $12.5 \mathrm{mg}(n=86$, gray bars). Data are shown for the patients of the intention-to-treat population with valid 24-h recordings $(n=181)$

(9.0, 2.4) mmHg; $P=0.969]$ and SBP [9.8 (14.4, $5.3)$ vs. $12.0(17.7,6.3) \mathrm{mmHg} ; P=0.561]$.

Assessment of the homogeneity of the BP control by the smoothness index, showed a comparable persistent antihypertensive efficacy of the two drugs over the $24 \mathrm{~h}$ for both DBP and SBP (Fig. 4).

\section{Effects on hs-CRP}

In total, 91 patients had evaluable blood samples for hs-CRP quantification. In the 51 patients treated with zofenopril plus diuretic, hs-CRP was reduced from $1.59 \pm 2.88$ to $1.40 \pm 2.03 \mathrm{mg} / \mathrm{L}$, while in the 40 patients treated with the irbesartan plus diuretic hsCRP remained stable during treatment (baseline $1.44 \pm 2.20 \mathrm{mg} / \mathrm{L}$; end of treatment $1.45 \pm 2.17 \mathrm{mg} / \mathrm{L}$ ). This resulted in a small baseline-adjusted reduction in hs-CRP by 
zofenopril $[-0.52(-1.05,0.01) \mathrm{mg} / \mathrm{L}]$ and in a slight increase by irbesartan $[0.97(0.29,1.65)$ $\mathrm{mg} / \mathrm{L}, P=0.001$ between treatments].

\section{Safety and Tolerability}

Laboratory and safety analyses were carried out in all randomized patients $(n=361)$. A total number of 88 (24.4\%) patients reported adverse events (48 in the zofenopril plus HCTZ and 40 in the irbesartan plus HCTZ treatment group): 126 adverse events ( 71 under zofenopril and 55 under irbesartan) were reported in at least 2 patients and most of them were of a mild intensity. Seven (1.9\%) patients were withdrawn from the study due to adverse events, all considered as drug related (4 in the zofenopril and 3 in the irbesartan group).

Events attributed to study treatment occurred in 26 patients (7.2\%), of which 14 $(7.8 \%)$ were treated with zofenopril plus the diuretic and 12 (6.6\%) with irbesartan plus the diuretic. A total of 24 drug-related adverse events were reported in at least two patients with a comparable distribution between the two study groups (10 zofenopril vs. 14 irbesartan). The most common drug-related adverse events observed under zofenopril were cough (4 cases), malaise (3 cases), and headache (2 cases), whereas dizziness (4 cases), asthenia (3 cases), abdominal pain (3 cases), and hypotension ( 2 cases) were more prevalent in irbesartan-treated patients. All these side effects may be expected with these classes of drugs.

Treatment was accompanied either by no change or only small and meaningless changes in the laboratory values considered in the study.

\section{DISCUSSION}

In hypertensive patients uncontrolled by a previous monotherapy and with additional cardiovascular risk factors, the office DBP reduction observed after 18 weeks of treatment with zofenopril 30 or $60 \mathrm{mg}$ plus HCTZ $12.5 \mathrm{mg}$ once daily was similar to that of irbesartan 150 or $300 \mathrm{mg}$ plus HCTZ $12.5 \mathrm{mg}$ once daily. This effect was obtained with the higher drug dosage in a slightly larger proportion of subjects treated with zofenopril (69\%) than with irbesartan (61\%), most likely because of a worse cardiovascular risk profile in the former group. Additionally, the antihypertensive effect of the zofenopril $30 \mathrm{mg}$ plus HCTZ $12.5 \mathrm{mg}$ combination was superior to that of the irbesartan $150 \mathrm{mg}$ plus HCTZ $12.5 \mathrm{mg}$ combination.

Approximately, $80 \%$ of patients attained the target $\mathrm{BP}$ of $<140 / 90 \mathrm{mmHg}, 60 \%$ that of $<130 / 80 \mathrm{mmHg}$, and $90 \%$ were classified as normalized or responders; such proportions were well balanced between the two drug treatment arms. These results strongly support that in the majority of patients not responding to a single antihypertensive medication, combination treatment with two drugs, including that between a drug acting on the angiotensin-renin-aldosterone system and a thiazide diuretic, may substantially increase the chance of response [33].

The BP lowering effect of both medications was well maintained also in subgroups of patients, such as diabetics, known to often show a reduced response to antihypertensive drug treatment [34]. This result, although limited to a small subgroup of 63 patients, also strongly supports that ACE inhibitors or ARBs are among the most suited first options in these high-risk patients [9].

The good office BP control obtained with zofenopril and irbesartan was confirmed over $24 \mathrm{~h}$ by ambulatory monitoring. Both drugs displayed a similarly smooth and long-lasting antihypertensive effect, ensuring a good BP 
coverage also in the hours farthest from the last drug intake [35]. Interestingly, the magnitude of the 24-h BP reduction yielded by zofenopril and irbesartan plus the diuretic in our study was comparable with that observed in previous studies based on ambulatory BP monitoring and making use of the same doses of the two drug combinations [36, 37].

This is the first study specifically assessing the antihypertensive efficacy of high-dose zofenopril (60 mg) plus HCTZ $12.5 \mathrm{mg}$. In previous studies, treatment with a 30- or $60-\mathrm{mg}$ dose of zofenopril combined with HCTZ $12.5 \mathrm{mg}$ once daily was superior to monotherapy with either agent $[25,36]$. In a dose-finding multifactorial study, the proportions of normalized and of normalized or responders to zofenopril plus HCTZ were $57 \%$ and $80 \%$, respectively, for the $30-\mathrm{mg}$ dose, and $79 \%$ and $93 \%$, for the $60-\mathrm{mg}$ dose $(80 \%$ and $88 \%$ in our study) [36]. In another study, administration of zofenopril $30 \mathrm{mg}$ and HCTZ $12.5 \mathrm{mg}$ combination for 8 weeks to 369 patients who were not responsive to zofenopril monotherapy resulted in an increase in the response rate up to $64 \%$ for DBP and $53 \%$ for SBP [26].

The benefits of the irbesartan and HCTZ combination therapy have been demonstrated in a number of trials in patients with mild hypertension [38-40]. These include the COSIMA [41] study and the INCLUSIVE [42] study, evaluating the efficacy of the combination in patients failing to achieve BP control with monotherapy. In such studies, 8-week treatment with a combination of irbesartan 150 or $300 \mathrm{mg}$ and HCTZ 12.5 or $25 \mathrm{mg}$ resulted in $50-70 \%$ of patients achieving BP normalization.

This is also the first study comparing zofenopril in combination with a thiazide diuretic with an ARB combined with a diuretic: previous direct comparative studies based on zofenopril monotherapy did not show any relevant difference in treatment efficacy vs. an ARB-based monotherapy regimen [21, 22]. The combination between irbesartan and a thiazide diuretic has also never been directly compared against that of an ACE inhibitor plus a diuretic, while evidence from comparative trials vs. an ACE-inhibitor monotherapy is available: in these studies irbesartan monotherapy at doses ranging between 75 and $300 \mathrm{mg}$ was as effective in lowering office BP and achieving BP control as enalapril [43-46] or fosinopril [47].

Our study also explored the possible positive effect of zofenopril and irbesartan on markers of vascular inflammation. As a matter of fact, the results of epidemiological and clinical studies support a pivotal role for inflammation in all phases of atherosclerosis, from endothelial cell dysfunction to the culmination in acute coronary syndrome [48]. In our hypertensive subjects, treatment with zofenopril plus HCTZ reduced hs-CRP, while this was not the case for irbesartan-treated patients. This is the first evidence of such an effect for zofenopril and it strongly supports previous findings in patients with hypertension that treatment with zofenopril may be associated with a reduction in oxidative stress, an improvement in the nitric oxide pathway, and an anti-inflammatory vasculoprotective effect [49]. The above results, although based on small numbers, allow speculating that the sulfhydryl ACE-inhibitor zofenopril, in addition to ensuring BP control, may contribute to slow down the development of atherosclerosis.

Both combination treatments were well tolerated, with a very limited number of drugrelated adverse events. As expected, the combination containing zofenopril was associated with a slightly larger incidence of 
cough, while dizziness or asthenia were more commonly reported by irbesartan-treated patients. Other adverse drug reactions were well balanced between the two groups and the overall tolerability profile of zofenopril and irbesartan was comparable with that in previous reports $[25,50,51]$.

The results of our study deserve some notes of caution. First, the sample size of patients with valid ambulatory BP recordings approximated $50 \%$ of that included in the primary study endpoint analysis. Unfortunately, this occurred because many recordings were missing or qualitatively inadequate. However, in the ambulatory BP monitoring subgroup, the effect of both drugs on office BP was comparable with that observed for the whole study population, indicating that the two populations were homogeneous. Second, the estimated standard deviation of the difference (9 $\mathrm{mmHg}$ ) may have been too large. However, a post hoc sample size calculation, using a smaller value $(5 \mathrm{mmHg})$ and retaining the same number of subjects, returned a power close to $100 \%$, thus confirming the appropriateness of our study sample size. Third, analyses on subgroups such as diabetics were not conceived at the time the study was planned. Consequently, we cannot exclude that our findings regarding such a subgroup might be the result of chance. Fourth, the population with valid hs-CRP assessment was smaller than the main study population, though the effect observed in zofenopril-treated patients for this inflammatory marker is in line with findings from previous studies [49]. We also attempted an exploratory analysis on patients with abnormal hs-PCR levels (>3 mg/L) $[52,53]$, but since only 11 patients fell in this group we could not obtain any reliable result. Finally, we did not foresee adjustment of randomization in the two groups by specific comorbidities, and since the two groups differed in several comorbid features at baseline we cannot exclude that some drug effects might be related to these factors. For these reasons we settled to adjust the comparison, whenever possible, for these source of unbalance.

\section{CONCLUSION}

The present pharmacological trial demonstrated that the combinations of zofenopril and HCTZ and that of irbesartan and HCTZ both provide similarly effective, prolonged and well-tolerated control of BP in hypertensive patients not controlled by previous monotherapy and with one or more additional cardiovascular risk factors. The reduction in hs-CRP observed with treatment with zofenopril plus the diuretic suggests an additional beneficial effect of this combination therapy on vascular inflammation.

\section{ACKNOWLEDGMENTS}

The present study was supported by a grant from Menarini International Operations Luxembourg S.A. Article processing charges were funded by Menarini International Operations Luxembourg S.A. S. Omboni is the guarantor for this article and takes responsibility for the integrity of the work as a whole. Study coordinators: E. Agabiti-Rosei (Italy), A. Manolis (Greece). List of study sites and investigators-Italy: F. Portaluppi (Ferrara), M. Santonastaso (Vittorio Veneto), M. Destro (Broni), G. Lembo (Pozzilli), A. Mezzetti (Chieti), P.L. Caroli (Lecce), A. Gargiulo (Caserta); Greece: A. Manolis (Athens), K. Tsioufis (Athens), V. Adamopoulou (Piraeus), V. Votteas (Athens); Lithuania: R. Kavaliauskiene (Klaipeda), G. Sakalyte 
(Kaunas), R. Steponéniené (Vilnius); Romania: L. Nedelcu (Brasov), A. Abaitancei (Brasov), L. Mathè (Tg. Mures), D. Nistorescu (Bucharest), D. Tint (Brasov); Turkey: C. Erol (Ankara), D. Ural (Izmit), F. Ozerkan (Izmir), T. Celik (Ankara), A. Abaci (Ankara), N. Baris (Izmir), S. Kucukoglu (Istanbul), B. Ilerigelen (Istanbul), S. Guneri (Izmir), M. Ozenci (Ankara).

Conflict of interest. E. Agabiti-Rosei has occasionally received grants for lectures or for attending scientific meetings by the manufacturers of zofenopril (Menarini International Operations Luxembourg S.A., Menarini Group). A. Manolis has occasionally received grants for lectures or for attending scientific meetings by the manufacturers of zofenopril. D. Zava is an employee of Istituto Lusofarmaco d'Italia, Menarini Group. S. Omboni has occasionally received grants for lectures or for attending scientific meeting by the manufacturers of zofenopril.

Compliance with ethics guidelines. The study was conducted according to Good Clinical Practice guidelines and the protocol was approved by the Ethics Committees of the centers involved. All procedures followed were in accordance with the ethical standards of the responsible committee on human experimentation (institutional and national) and with the Helsinki Declaration of 1975, as revised in 2000 and 2008. Informed consent was obtained from all patients for being included in the study.

Open Access. This article is distributed under the terms of the Creative Commons Attribution Noncommercial License which permits any noncommercial use, distribution, and reproduction in any medium, provided the original author(s) and the source are credited.

\section{REFERENCES}

1. Kearney PM, Whelton M, Reynolds K, Muntner P, Whelton PK, He J. Global burden of hypertension: analysis of worldwide data. Lancet. 2005;365:217-23.

2. Lewington S, Clarke R, Qizilbash N, Peto R, Collins $\mathrm{R}$, Prospective Studies Collaboration. Age-specific relevance of usual blood pressure to vascular mortality: a meta-analysis of individual data for one million adults in 61 prospective studies. Lancet. 2002;360:1903-13.

3. Neal B, MacMahon S, Chapman N, Blood Pressure Lowering Treatment Trialists' Collaboration. Effects of ACE inhibitors, calcium antagonists, and other blood-pressure-lowering drugs: results of prospectively designed overviews of randomised trials. Blood Pressure Lowering Treatment Trialists' Collaboration. Lancet. 2000;356:1955-64.

4. Turnbull F, Blood Pressure Lowering Treatment Trialists' Collaboration. Effects of different bloodpressure-lowering regimens on major cardiovascular events: results of prospectivelydesigned overviews of randomised trials. Lancet. 2003;362:1527-35.

5. The Heart Outcome Prevention Evaluation Study Investigators. Effects of an angiotensin-convertingenzyme inhibitor, ramipril, on cardiovascular events in high-risk patients. $\mathrm{N}$ Engl J Med. 2000;342:145-53.

6. PROGRESS Collaborative Study Group. Randomised trial of perindopril based blood pressure-lowering regimen among 6108 individuals with previous stroke or transient ischaemic attack. Lancet. 2001;358:1033-41.

7. The ALLHAT Officers and Coordinators for the ALLHAT Collaborative Research Group. Major outcomes in high-risk hypertensive patients randomized to angiotensin-converting enzyme inhibitor or calcium channel blocker vs diuretic: the Antihypertensive and Lipid-Lowering treatment to prevent Heart Attack Trial (ALLHAT). JAMA. 2002;288:2981-97.

8. Julius S, Kjeldsen SE, Weber M, et al. Outcomes in hypertensive patients at high cardiovascular risk treated with regimens based on valsartan or amlodipine: the VALUE randomised trial. Lancet. 2004;363:2022-31.

9. Mancia G, Fagard R, Narkiewicz K, et al. 2013 ESH/ ESC guidelines for the management of arterial hypertension: the task force for the management of arterial hypertension of the European Society of 
Hypertension (ESH) and of the European Society of Cardiology (ESC). J Hypertens. 2013;31:1281-357.

10. Chobanian AV, Bakris GL, Black HR, Cushman WC, Green LA, Izzo JL Jr, Jones DW, Materson BJ, Oparil S, Wright Jr JT, Roccella EJ, National Heart, Lung, and Blood Institute Joint National Committee on Prevention, Detection, Evaluation, and Treatment of High Blood Pressure; National High Blood Pressure Education Program. The seventh report of the Joint National Committee on Prevention, Detection, Evaluation, and Treatment of High Blood Pressure: the JNC 7 report. JAMA. 2003;289:2560-72.

11. Waeber B. Combination therapy with ACE inhibitors/angiotensin II receptor antagonists and diuretics in hypertension. Expert Rev Cardiovasc Ther. 2003;1:43-50.

12. Ambrosioni E. Defining the role of zofenopril in the management of hypertension and ischemic heart disorders. Am J Cardiovasc Drugs. 2007;7:17-24.

13. Borghi C, Bacchelli S, Degli Esposti D. Long-term clinical experience with zofenopril. Expert Rev Cardiovasc Ther. 2012;10:973-82.

14. Borghi C, Ambrosioni E, Omboni S, et al. Zofenopril and ramipril and acetylsalicylic acid in postmyocardial infarction patients with left ventricular systolic dysfunction: a retrospective analysis in hypertensive patients of the SMILE-4 study. J Hypertens. 2013;31:1256-64.

15. Borghi C, Bacchelli S, Esposti DD, Bignamini A, Magnani B, Ambrosioni E. Effects of the administration of an angiotensin-converting enzyme inhibitor during the acute phase of myocardial infarction in patients with arterial hypertension. SMILE Study Investigators. Survival of myocardial infarction long-term evaluation. Am J Hypertens. 1999;12:665-72.

16. Nilsson P. Antihypertensive efficacy of zofenopril compared with atenolol in patients with mild to moderate hypertension. Blood Press. 2007;2(Suppl):25-30.

17. Lacourciere Y, Provencher P. Comparative effects of zofenopril and HCTZ on office and ambulatory blood pressures in mild to moderate essential hypertension. Br J Clin Pharmacol. 1989;27:371-6.

18. Farsang C. Blood pressure control and response rates with zofenopril compared with amlodipine in hypertensive patients. Blood Press. 2007;2(Suppl): 19-24.

19. Mallion JM. An evaluation of the initial and longterm antihypertensive efficacy of zofenopril compared with enalapril in mild to moderate hypertension. Blood Press. 2007;2(Suppl):13-8.
20. Malacco E, Piazza S, Omboni S, Zofenopril Study Group. Zofenopril versus lisinopril in the treatment of essential hypertension in elderly patients: a randomised, double-blind, multicentre study. Clin Drug Investig. 2005;25:175-82.

21. Narkiewicz K. Comparison of home and office blood pressure in hypertensive patients treated with zofenopril or losartan. Blood Press. 2007;2(Suppl):7-12.

22. Leonetti G, Rappelli A, Omboni S, on behalf of the study group. A similar 24-h blood pressure control is obtained by zofenopril and candesartan in primary hypertensive patients. Blood Press. 2006;15(Suppl 1):18-26.

23. Borghi C, Cicero AF. The role of irbesartan in the treatment of patients with hypertension: a comprehensive and practical review. High Blood Press Cardiovasc Prev. 2012;19:19-31.

24. Bramlage P, Durand-Zaleski I, Desai N, Pirk O, Hacker C. The value of irbesartan in the management of hypertension. Expert Opin Pharmacother. 2009;10:1817-31.

25. Omboni S, Malacco E, Parati G. Zofenopril plus hydrochlorothiazide fixed combination in the treatment of hypertension and associated clinical conditions. Cardiovasc Ther. 2009;27:275-88.

26. Zanchetti A, Parati G, Malacco E. Zofenopril plus hydrochlorothiazide: combination therapy for the treatment of mild to moderate hypertension. Drugs. 2006;66:1107-15.

27. Mancia G, De Backer G, Dominiczak A, et al. 2007 Guidelines for the management of arterial hypertension: the task force for the management of arterial hypertension of the European Society of Hypertension (ESH) and of the European Society of Cardiology (ESC). J Hypertens. 2007; 25:1105-87.

28. Rogoza AN, Pavlova TS, Sergeeva MV. Validation of A\&D UA-767 device for the self-measurement of blood pressure. Blood Press Monit. 2000;5:227-31.

29. Palatini P, Frigo G, Bertolo O, Roman E, Da Cortà R, Winnicki M. Validation of the A\&D TM-2430 device for ambulatory blood pressure monitoring and evaluation of performance according to subjects' characteristics. Blood Press Monit. 1998;3:255-60.

30. MOREPRESS website. Monitoring remotely blood pressure. http://www.morepress.net. Accessed December 20, 2013.

31. Parati G, Omboni S, Rizzoni D, Agabiti-Rosei E, Mancia G. The smoothness index: a new, 
reproducible and clinically relevant measure of the homogeneity of the blood pressure reduction with treatment for hypertension. J Hypertens. 1998;16:1685-91.

32. Parati G, Omboni S, Palatini P, et al. Italian society of hypertension guidelines for conventional and automated blood pressure measurement in the office, at home and over 24 hours. High Blood Press Cardiovasc Prev. 2008;15:283-310.

33. Sica DA. Rationale for fixed-dose combinations in the treatment of hypertension: the cycle repeats. Drugs. 2002;62:443-62.

34. Working Party of the International Diabetes Federation (European Region). Hypertension in people with type 2 diabetes: knowledge-based diabetes-specific guidelines. Diabet Med. 2003;20:972-87.

35. Elliot WJ. Cyclic and circadian variations in cardiovascular events. Am J Hypertens. 2001;14(9 Pt 2):291S-5S.

36. Parati G, Omboni S, Malacco E. Antihypertensive efficacy of zofenopril and hydrochlorothiazide combination on ambulatory blood pressure. Blood Press. 2006;15(Suppl 1):7-17.

37. Neutel JM, Smith D. Ambulatory blood pressure comparison of the anti-hypertensive efficacy of fixed combinations of irbesartan/hydrochlorothiazide and losartan/hydrochlorothiazide in patients with mild-to-moderate hypertension. J Int Med Res. 2005;33:620-31.

38. Schrader J, Bramlage $P$, Lüders $S$, Thoenes $M$, Schirmer A, Paar DW. BP goal achievement in patients with uncontrolled hypertension: results of the treat-to-target post-marketing survey with irbesartan. Clin Drug Investig. 2007;27:783-96.

39. Kintscher $U$, Bramlage $P$, Paar WD, Thoenes $M$, Unger $T$. Irbesartan for the treatment of hypertension in patients with the metabolic syndrome: a sub analysis of the treat to target post authorization survey. Prospective observational, two armed study in 14,200 patients. Cardiovasc Diabetol. 2007;6:12.

40. Sun NL, Jing S, Chen J. The control rate of irbesartan/hydrochlorothiazide combination regimen in the treatment of Chinese patients with mild to moderate hypertension. Zhonghua Xin Xue Guan Bing Za Zhi. 2005;33:618-21.

41. Bobrie G, Delonca J, Moulin C, Giacomino A, Postel-Vinay N, Asmar R, COmparative Study of Efficacy of Irbesartan/HCTZ with Valsartan/HCTZ Using Home Blood Pressure Monitoring in the TreAtment of Mild-to-Moderate Hypertension
(COSIMA) Investigators. A home blood pressure monitoring study comparing the antihypertensive efficacy of two angiotensin II receptor antagonist fixed combinations. Am J Hypertens. 2005;18:1482-8.

42. Neutel JM, Saunders E, Bakris GL, et al. The efficacy and safety of low- and high-dose fixed combinations of irbesartan/hydrochlorothiazide in patients with uncontrolled systolic blood pressure on monotherapy: the INCLUSIVE trial. J Clin Hypertens (Greenwich). 2005;7:578-86.

43. Coca A, Calvo C, García-Puig J, et al. A multicenter, randomized, double-blind comparison of the efficacy and safety of irbesartan and enalapril in adults with mild to moderate essential hypertension, as assessed by ambulatory blood pressure monitoring: the MAPAVEL Study (Monitorización Ambulatoria Presión Arterial APROVEL). Clin Ther. 2002;24:126-38.

44. Lacourcière Y. A multicenter, randomized, doubleblind study of the antihypertensive efficacy and tolerability of irbesartan in patients aged > or $=65$ years with mild to moderate hypertension. Clin Ther. 2000;22:1213-24.

45. Chiou KR, Chen CH, Ding PY, et al. Randomized, double-blind comparison of irbesartan and enalapril for treatment of mild to moderate hypertension. Zhonghua Yi Xue Za Zhi (Taipei). 2000;63:368-76.

46. Mimran A, Ruilope L, Kerwin L, et al. A randomised, double-blind comparison of the angiotensin II receptor antagonist, irbesartan, with the full dose range of enalapril for the treatment of mild-tomoderate hypertension. J Hum Hypertens. 1998;12:203-8.

47. Angulo E, Robles NR, Grois J, Barquero A, Pérez Miranda M. Comparison of the antihypertensive activity of fosinopril and irbesartan (in Spanish). An Med Interna. 2002;19:571-5.

48. Jialal I, Devaraj S, Venugopal SK. C-reactive protein: risk marker or mediator in atherothrombosis? Hypertension. 2004;44:6-11.

49. Napoli C, Sica V, de Nigris F, et al. Sulfhydryl angiotensin-converting enzyme inhibition induces sustained reduction of systemic oxidative stress and improves the nitric oxide pathway in patients with essential hypertension. Am Heart J. 2004; 148:e5.

50. Bramlage P. Fixed combination of irbesartan and hydrochlorothiazide in the management of hypertension. Vasc Health Risk Manag. 2009;5:213-24. 
51. Forni V, Wuerzner G, Pruijm M, Burnier M. Longterm use and tolerability of irbesartan for control of hypertension. Integr Blood Press Control. 2011;4:17-26.

52. Haverkate F, Thompson SG, Pyke SD, et al. Production of C-reactive protein and risk of coronary events in stable and unstable angina. European Concerted Action on Thrombosis and Disabilities Angina Pectoris Study Group. Lancet. 1997;349:462-6.
53. Rebuzzi AG, Quaranta G, Liuzzo G, et al. Incremental prognostic value of serum levels of troponin $\mathrm{T}$ and $\mathrm{C}$-reactive protein on admission in patients with unstable angina pectoris. Am J Cardiol. 1998;82:715-9. 